\title{
Tunnel Magnetoresistance for Phonon-Assisted Tunneling through Quantum Dots
}

\author{
W. RUDZIŃSKI \\ Department of Physics, Adam Mickiewicz University \\ Umultowska 85, 61-614 Poznań, Poland
}

\begin{abstract}
Spin-polarized transport through a single-level quantum dot interacting with a local phonon mode is studied in the frame of the nonequilibrium Green-function technique. It is shown that strong electron-phonon interaction gives rise to a significant current suppression, to additional resonance peaks in the differential conductance as well as to oscillations of the tunnel magnetoresistance. Also, a phonon-induced mechanism leading to a minimum in tunnel magnetoresistance at zero bias voltage is explained.
\end{abstract}

PACS numbers: 72.25.Mk, 73.63.Kv, 73.23.Hk

\section{Introduction}

Electronic transport through discrete levels of single molecules, carbon nanotubes or suspended quantum dots (QDs) coupled to vibrational degrees of freedom has been investigated recently in a number of experimental as well as theoretical works (see e.g. [1-3]). The present work extends studies on polaronic transport in mesoscopic systems to the case of tunneling through a single-level interacting quantum dot coupled to ferromagnetic electrodes. One of the most widely studied spin-dependent effects in such magnetic tunnel junctions is the tunnel magnetoresistance (TMR) defined quantitatively as $\mathrm{TMR}=\left[J_{\mathrm{P}}-J_{\mathrm{AP}}\right] / J_{\mathrm{AP}}$, with $J_{\mathrm{P}}$ and $J_{\mathrm{AP}}$ denoting electric current in the parallel (P) and antiparallel (AP) magnetic configuration of the external electrodes, respectively.

\section{Model}

In the model Hamiltonian of the system, the left (l) and right (r) ferromagnetic electrodes are taken in the noninteracting quasiparticle limit, $\sum_{\nu, k, \sigma} \varepsilon_{k \sigma}^{\nu} a_{\nu k \sigma}^{+} a_{\nu k \sigma}$, where $\varepsilon_{k \sigma}^{\nu}$ is the single-electron energy in the $\nu$-th $(\nu=1, \mathrm{r})$ electrode for the wave vector $k$ and spin $\sigma(\sigma=\uparrow, \downarrow)$. The term corresponding to the dot includes the single particle energy level $\varepsilon_{d}$ as well as the Coulomb correlations 
described by the Hubbard parameter $U, \sum_{\sigma} \varepsilon_{d} c_{\sigma}^{+} c_{\sigma}+U n_{\uparrow} n_{\downarrow}$. The phonon Hamiltonian includes the quantity $\omega_{0}$ describing the vibrational frequency of the phonon mode and the electron-phonon interaction term with the parameter $\lambda$ denoting the strength of the electron-phonon coupling, $\omega_{0} b^{+} b+\sum_{\sigma} \lambda\left(b+b^{+}\right) c_{\sigma}^{+} c_{\sigma}$. Finally, the tunneling part includes spin-dependent tunneling processes, $\sum_{\nu, k, \sigma} T_{k \sigma}^{\nu} a_{\nu k \sigma}^{+} c_{\sigma}+$ h.c., where $T_{k \sigma}^{\nu}$ is the tunneling amplitude. Spin asymmetry of the tunneling rates across the left and right barriers is described by the parameters $p_{1}$ and $p_{\mathrm{r}}$. To calculate the density matrix for the system we used nonequilibrium Green-function method based on equation of motion in the Hartree-Fock approximation (see e.g. [4]). The assumed strong electron-phonon interaction regime allowed us to eliminate the linear electron-phonon coupling terms in the phonon Hamiltonian by using the Lang-Firsov-type unitary transformation [5]. Consequently, the electron part is reshaped to the standard form of the Anderson Hamiltonian with renormalized energy of the dot discrete level, $\varepsilon_{d}^{\prime}=\varepsilon_{d}-\lambda^{2}$ and renormalized Coulomb charging energy, $U^{\prime}=U-2 \lambda^{2}$. The tunneling amplitudes are also renormalized, describing the fact that the electron hopping is exponentially suppressed with increasing parameter $\lambda$. Having found self-consistently the occupation numbers, one can calculate the tunneling current from the Meir-Wingreen formula [6].

\section{Numerical results}

Let us discuss features of the polaronic transport through symmetrical tunnel junctions, i.e. assume that both barriers are identical and $p_{1}=p_{\mathrm{r}}$. The effective dot energy level is assumed to be empty in the equilibrium situation, $\varepsilon_{d}^{\prime}>0$ at $V=0$. Moreover, for clarity of our presentation, the electric current as well as the corresponding conductance will be shown only for the $\mathrm{P}$ magnetic configuration. The contribution from the AP configuration is always present in the displayed TMR ratios.

As shown in Fig. 1a, for a positive effective charging energy, $U^{\prime}>0$, and in the presence of strong electron-phonon interactions the exponential suppression of the tunneling rates gives rise to a significant suppression of the electric current in the whole bias voltage range (see the inset in Fig. 1a for details). Since tunneling processes may be mediated by the phonon energy levels, then besides the two steps in the current at which $\varepsilon_{d}^{\prime}$ and $\varepsilon_{d}^{\prime}+U^{\prime}$ enter the tunneling window, also additional Frank-Condon steps appear. Hence, phonon emission at $T=0$ gives rise to vibrational side bands in the differential conductance (dotted line in Fig. 1a), spaced at the phonon energy from the two elastic resonance peaks. The corresponding TMR exhibits oscillations above the bias voltage region, where the sequential tunneling current is exponentially suppressed. The amplitude of these oscillations increases due to spin accumulation on the dot, which occurs between each pair of phonon resonances.

If the polaron shift leads to a negative effective charging energy, then it is known [6] that in the Coulomb blockade regime ground states with even numbers 


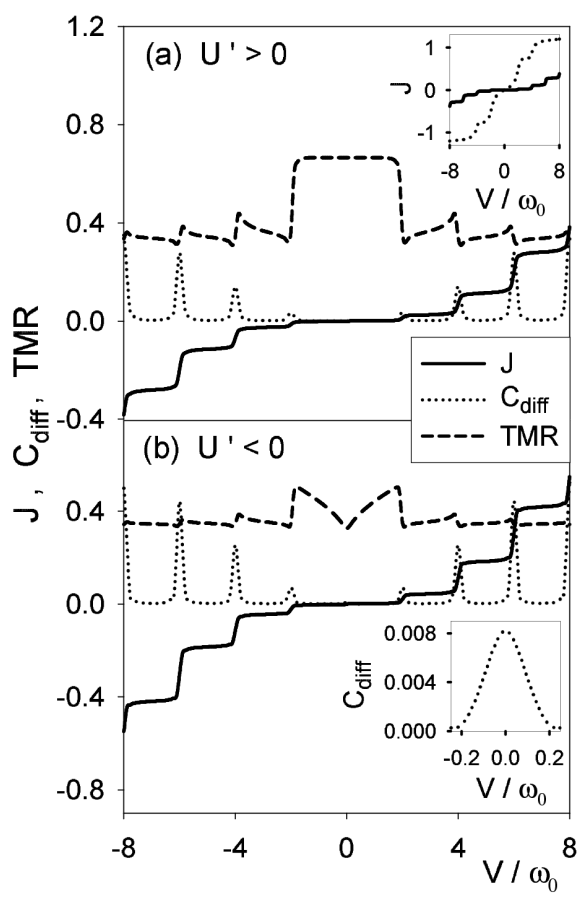

Fig. 1. Bias dependence of the electric current $J$, differential conductance $C_{\text {diff }}$ in $\mathrm{P}$ configuration and the corresponding TMR for (a) $U^{\prime}=1$ and (b) $U^{\prime}=-9$. The other parameters are $p_{1}=p_{\mathrm{r}}=0.5, \varepsilon_{d}^{\prime}=1, \lambda=1$ and $T=0$. The energy is measured relative to the phonon excitation energy $\omega_{0}$. The dotted and solid curves in the inset in (a) show the difference between the electric current for the system without $(\lambda=0)$ and with $(\lambda=1)$ electron-phonon interactions, respectively. The inset in (b) shows an enlargement of the conductance peak at the zero bias voltage, occurring for the case of negative $U^{\prime}$.

of electrons are favored and thus in parallel with single-particle cotunneling, sequential pair-tunneling processes also take place. To be more precise, at finite $U^{\prime}<0$ two electrons can hop onto the dot simultaneously from (or out to) opposite leads and charge transport may be dominated by tunneling of electron pairs. As shown in Fig. 1b such pair tunneling leads to a conductance peak at zero bias voltage. In magnetic tunnel junctions this feature results also in a remarkable minimum of TMR at $V=0$. Thus, the visible in Fig. 1a central maximum of TMR originating from the single-electron higher-order tunneling processes is now split due to pair-tunneling into two TMR maxima.

\section{Conclusions}

The present work reveals that for strong enough electron-phonon coupling the exponential suppression of tunneling rates gives rise to step-like electric current 
behavior with additional Franck-Condon steps appearing at threshold bias voltages at which phonon-assisted electron transmission takes place. Consequently, oscillations of the corresponding TMR have been observed in the nonlinear response regime. When the polaron shift induces a negative effective charging energy, then a competition between the single-electron cotunneling and pair-tunneling processes leads to a splitting of the TMR maximum in the Coulomb blockade regime.

\section{Acknowledgments}

This work was supported by funds of the Polish Ministry of Science and Higher Education as a research project in years 2006-2009.

\section{References}

[1] H. Park, J. Park, A. K.L. Lim, E.H. Anderson, A.P. Alivisatos, P.L. McEuen, Nature 407, 57 (2000).

[2] M. Galperin, A. Nitzan, M.A. Ratner, Phys. Rev. B 73, 045314 (2006).

[3] J. Koch, M.E. Raikh, F. von Oppen, Phys. Rev. Lett. 96, 056803 (2006).

[4] W. Rudziński, J. Barnaś, R.Świrkowicz, M. Wilczyński, Phys. Rev. B 71, 205307 (2005).

[5] G.D. Mahan, Many-Particle Physics, Plenum Press, New York 2000.

[6] A.-P. Jauho, N.S. Wingreen, Y. Meir, Phys. Rev. B 50, 5528 (1994). 